\title{
Minimally Invasive Surgery for Intracochlear Schwannoma Removal and Simultaneous Cochlear Implantation
}

\author{
Ricardo Ferreira Bento ${ }^{1}$ Eloisa Maria Mello Santiago Gebrim² ${ }^{2}$ Ana Tereza de Matos Magalhães ${ }^{1}$ \\ Larissa Vilela Pereira ${ }^{1}$ Anna Carolina de Oliveira Fonseca ${ }^{1}$ \\ ${ }^{1}$ Department of Otolaryngology, School of Medicine, Universidade de \\ São Paulo, São Paulo, SP, Brazil \\ 2 Department of Radiology, School of Medicine, Universidade de São \\ Paulo, São Paulo, SP, Brazil \\ Address for correspondence Ricardo Ferreira Bento, MD, PhD, \\ Department of Otorhinolaryngology, HCFMUSP, Rua Dr. Ovídio Pires \\ de Campos, 225 - Cerqueira César, São Paulo SP 05403-010, Brazil \\ (e-mail: rbento@gmail.com).
}

Int Arch Otorhinolaryngol 2016;20:271-274.

\begin{abstract}
Introduction Hearing preservation has not yet been reported in patients undergoing resection of intracochlear schwannomas. This study describes a minimally invasive procedure for intracochlear schwannoma resection with simultaneous cochlear implantation that resulted in good hearing.

Objective This study aims to describe a minimally invasive procedure for intracochlear schwannoma resection with simultaneous cochlear implantation.

Data Synthesis The technique described in this study was developed for a 55 -year-old male with a 20-year history of bilateral progressive hearing loss and tinnitus that had a mass in the left apical turn of the cochlea measuring $0.3 \mathrm{~cm}$. Surgery accessed the apical turn of the cochlea. We performed mastoidectomy and posterior tympanotomy and removed incus and tensor tympani muscle to expose the cochlear apex. The tumor was identified and completely resected. After the cochlea was anatomically preserved, it was

Keywords

- cochlea

- intracochlear schwannoma

- hearing loss

- hearing rehabilitation

- cochlear implantation implanted with a straight electrode via round window insertion. The histopathological examination confirmed intracochlear schwannoma. Speech perception test revealed $100 \%$ speech recognition with closed sentences and the average audiometric threshold (500 to $2000 \mathrm{~Hz}$ ) was $23 \mathrm{~dB}$.

Conclusion Our technique led to rehabilitation of the patient and improved hearing without damaging the intracochlear structure, making it possible to perform $\mathrm{Cl}$ in the same procedure with good results.
\end{abstract}

\section{Introduction}

Minimally invasive surgeries are more common in today's clinical practice. The cochlea is a particularly differentiated organ with delicate microscopic architecture that is easily damaged during surgical procedures. Access to the cochlea through the internal ear was taboo for surgeons until the stapedotomy approach was introduced by Shea. With the

received

October 19, 2015

accepted

February 12, 2016

published online

April 7, 2016
DOI http://dx.doi.org/

10.1055/s-0036-1581091. ISSN 1809-9777. advent of cochlear implant $(\mathrm{CI})$ surgery, the barrier to cochlear surgery was broken. CI surgery has been performed for more than 30 years and is the standard procedure for restoring hearing in patients with severeto-profound hearing loss. $\mathrm{Cl}$ indications have expanded to allow for the restoration of moderate-to-severe hearing loss. For this purpose, preservation of the cochlear architecture is essential. ${ }^{1}$
Copyright $\odot 2016$ by Thieme Publicações License terms Ltda, Rio de Janeiro, Brazil

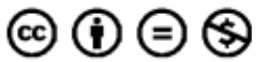


Von Ilberg et $\mathrm{al}^{2}$ first described a cochlear surgery that preserved residual hearing. Substantial progress has been made in cochlear surgical techniques, including the development of atraumatic cochleostomy and round window electrode insertion and the use of intraoperative lubricant solutions, which improved the ability to slide the electrode into the cochlea. Additionally, new implants are continuously being developed, and the improvements in electrode design have facilitated atraumatic surgery with the aim of preserving residual hearing. ${ }^{1}$ Skarzynski et $\mathrm{al}^{3}$ and Bento et $\mathrm{al}^{4}$ have demonstrated that residual hearing can be preserved during $\mathrm{Cl}$ surgery.

Progress in cochlear implant surgical techniques and technology have brought a new interest in bimodal stimulation. Now, specific frequencies can be stimulated to improve cochlear tonotopy. Electroacoustic implants allow for bimodal stimulation. High frequencies are electrically stimulated, and low frequencies are acoustically amplified. The bimodal stimulation results in less noise and improved musical perception. ${ }^{5}$

Intralabyrinthine schwannomas (ILS) are tumors that primarily arise from within the membranous labyrinth (the cochlea, vestibule, and semi-circular canals). To date, only 137 cases have been reported. ${ }^{6}$ Salzman et al presented an anatomically based classification system for ILS, including intravestibular, intracochlear, vestibulocochlear, transmodiolar, transmacular, and transotic. Intracochlear schwannomas (ICS) are the dominant ILS type according to this classification system. The treatment of ICS depends on tumor size and growth. ${ }^{6}$ The degree of hearing loss, the presence of vestibular symptoms, and the pathologic diagnosis must also be considered. ${ }^{7}$

Hearing preservation has not yet been reported in patients undergoing resection of intracochlear schwannomas. Anacusis often occurs as a result of the natural history of the tumor or after surgical removal. ${ }^{8}$

This study describes a minimally invasive procedure for intracochlear schwannoma resection with simultaneous cochlear implantation that resulted in good hearing.

To our knowledge, we are the first to report an ICS with a $\mathrm{Cl}$ during the same surgery procedure and that is our report's main contribution. Kronenberg et al do report an ICS with a CI, but their ICS was incidentally discovered during surgery and the $\mathrm{Cl}$ performed three years later, whereas ours was previously diagnosed and planned to perform during the same surgery. The results of the patients auditory speech perception were fairly good. The score was 100\% in closed set and 30\% in open set presentation of topic-related, everyday sentences. ${ }^{9}$

\section{Review of a Particular Subject}

The technique described in this study was developed for a patient with a 20-year history of bilateral progressive hearing loss and tinnitus. The patient was an otolaryngologist and was hindered from working in his chosen profession due to hearing loss and incapacitating vertigo. His hearing had gradually deteriorated on the left side, resulting in profound hearing loss. The patient also developed vertigo. Magnetic resonance imaging (MRI) with gadolinium revealed a mass in the left apical turn of the cochlea measuring $0.3 \mathrm{~cm}$ (-Figs. 1, 2, and 3).

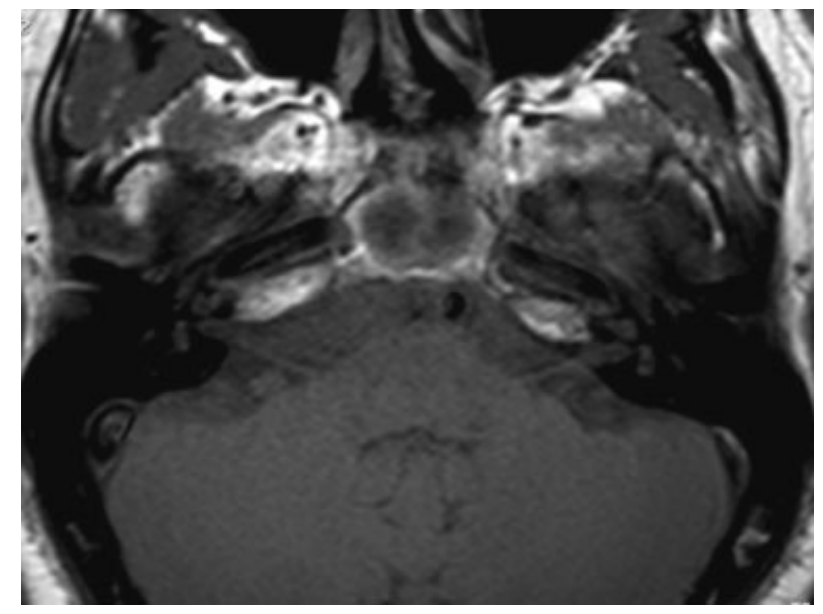

Fig. 1 Pre-gadolinium T1.

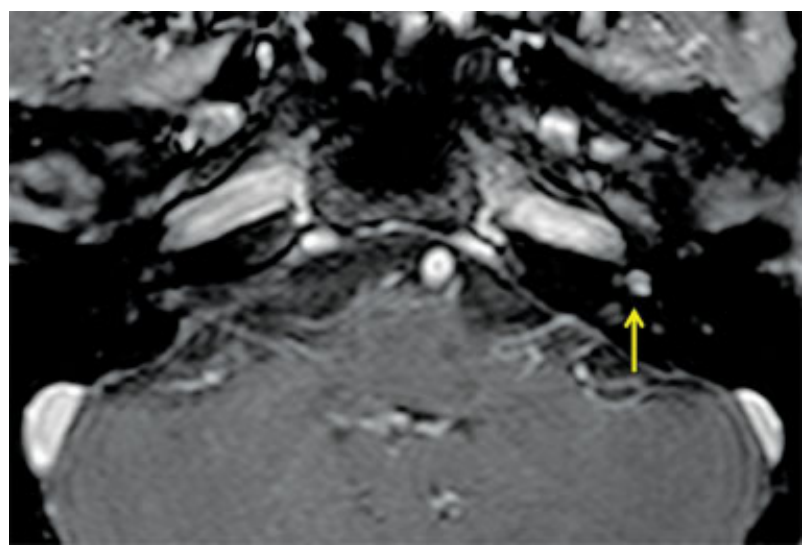

Fig. 2 Post-gadolinium T1, enhanced nodular mass in the left apical turn of the cochlea measuring $0.3 \mathrm{~cm}$.

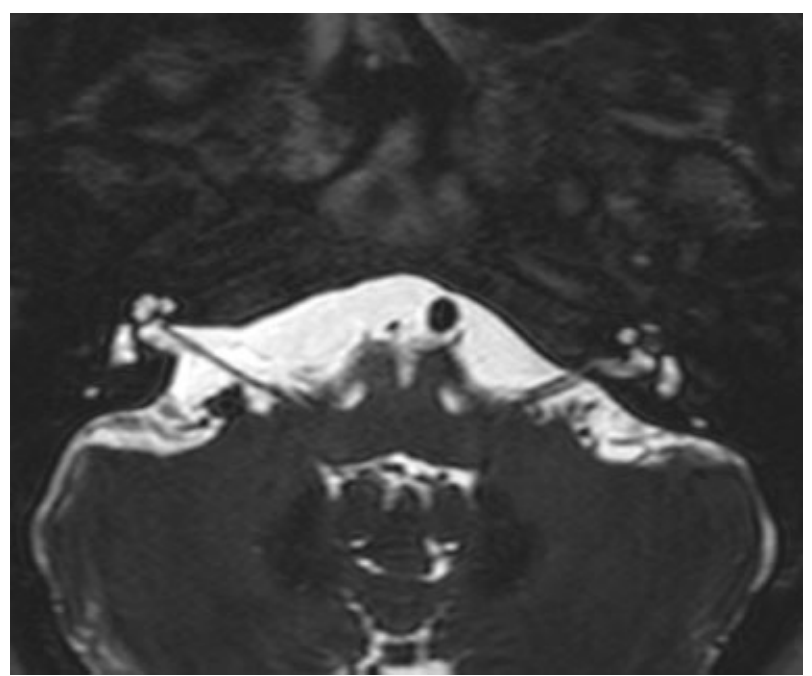

Fig. 3 T2, Low signal mass in the left apical turn of the cochlea.

His hearing had also deteriorated on the right side, and his hearing aid (HA) was still providing some benefit to the right ear (in contrast to the left side) - Fig. 4. demonstrates his audiometry with bilateral sensorineural hearing loss, severe at the right side and profound at the left side. 


\section{Audiometry}

Right Ear

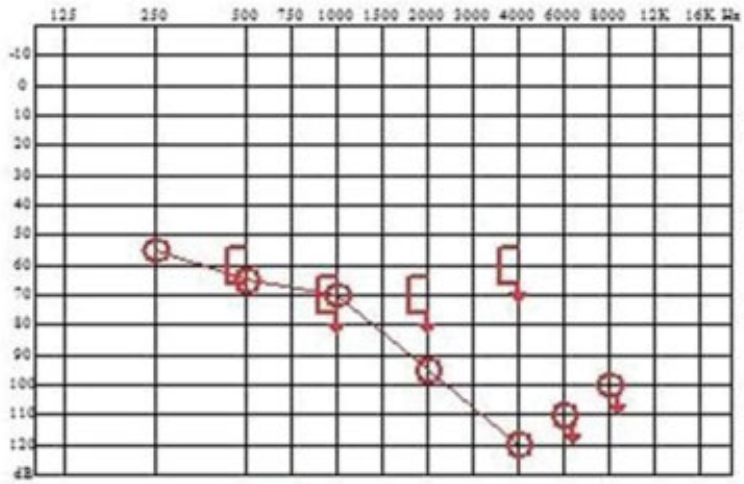

SRT: $75 \mathrm{~dB}$
Left Ear

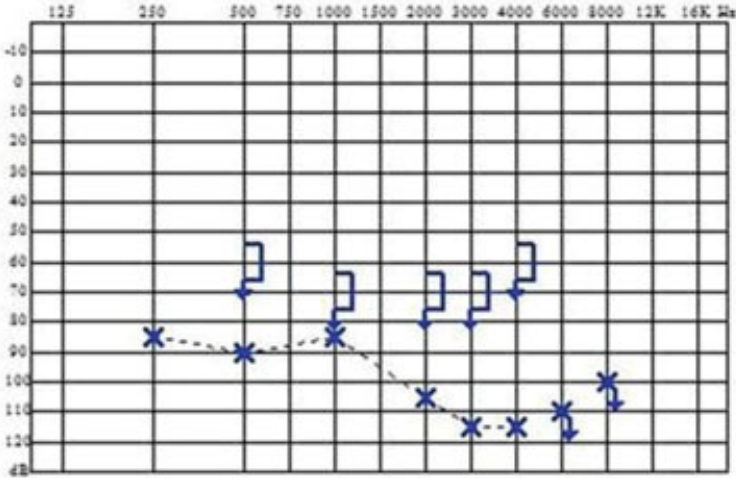

SRT:

Speech Audiometry

\begin{tabular}{|l|l|l|l|}
\hline & Intensity & Monosyllable & Dissyllable \\
\hline Right Ear & $85 \mathrm{~dB}$ & $76 \%$ & $80 \%$ \\
\hline Left Ear & $95 \mathrm{~dB}$ & $0 \%$ & $0 \%$ \\
\hline
\end{tabular}

Fig. 4 Audiometry with bilateral sensorineural hearing loss, severe at the right side and profound at the left side.

This surgery accessed the apical turn of the cochlea. We performed mastoidectomy and posterior tympanotomy, removing the incus and tensor timpani muscle to expose the cochlear apex. The cochlear wall was burred with a small diamond burr. A small window in the apical turn was opened, and the membranous labyrinth was exposed.

The opening was enlarged with a delicate curette. The tumor was identified, pulled out with a delicate hook, and completely resected (-Figs. $\mathbf{5}$ and $\mathbf{6}$ ).

The small window in the apical turn was sealed with bone wax. After the cochlea was anatomically preserved, it was

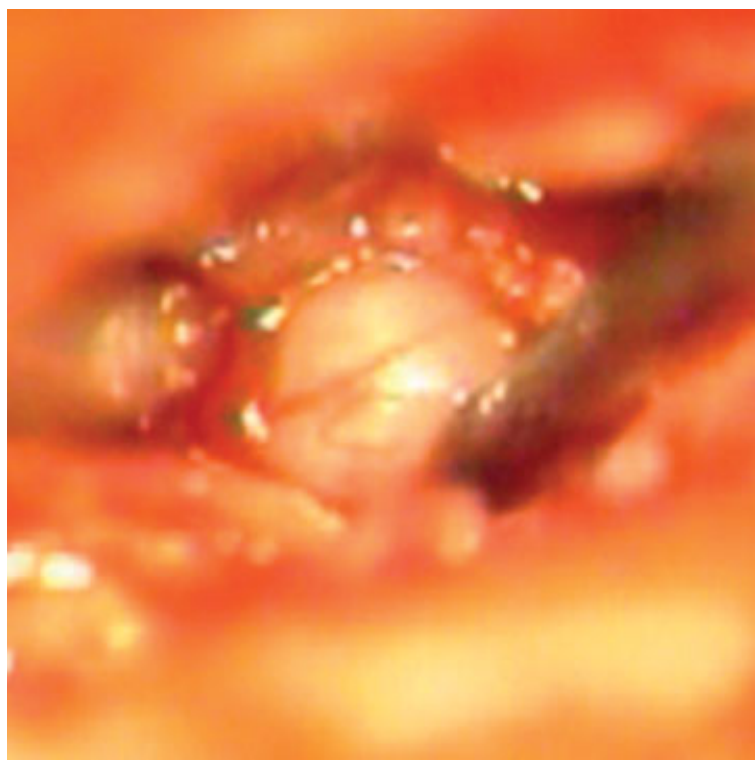

Fig. 5 Apical turn of cochlea exposed with tumor inside. implanted with a straight electrode (Nucleus CI422) via round window insertion. Computed tomography scans indicated complete electrode insertion into the cochlea. The histopathological examination confirmed intracochlear schwannoma.

We performed tumor resection to treat the patient's vertigo, and performed simultaneous $\mathrm{CI}$ implantation to restore his hearing. Immediately after surgery, the patient's vertigo and dizziness resolved and the tinnitus improved. A speech processor was activated four weeks after implantation. All electrodes (22/22) were used for electric stimulation, and neural response telemetry indicated that all electrodes were functional. After three months of speech processor use, an auditory speech perception test revealed $100 \%$ speech recognition with closed sentences, and the average

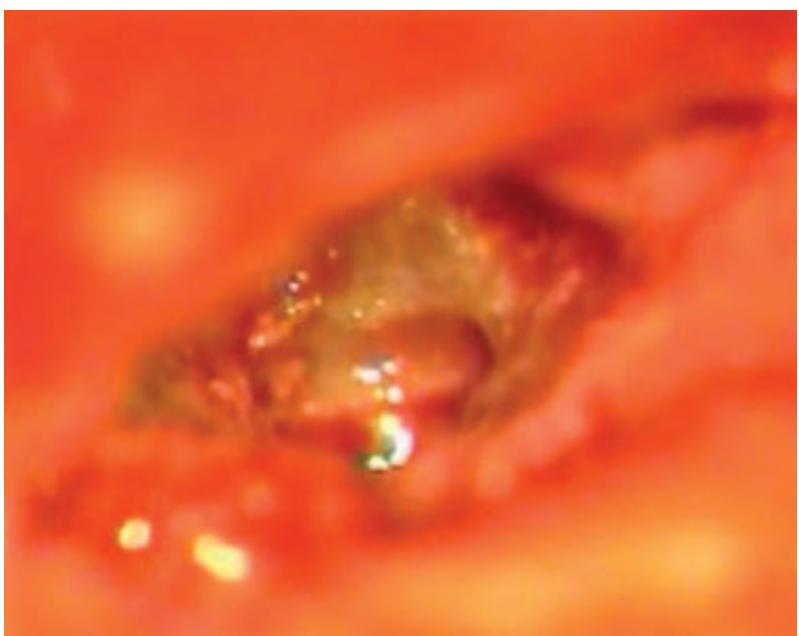

Fig. 6 Apical turn of the cochlea exposed following tumor removal. 
audiometric threshold (500 to $2000 \mathrm{~Hz}$ ) was $23 \mathrm{~dB}$. The patient continues to use a HA in the contralateral ear and his speech perception with open sentences is $70 \%$ using only the HA. However, with bimodal stimulation $(\mathrm{HA}+\mathrm{CI})$, the score is $100 \%$, which indicates that $\mathrm{CI}$ improved this patient's speech recognition.

Radiological examinations during the five follow-up years did not indicate tumor growth.

\section{Discussion}

With the recent improvements in imaging techniques, an increasing number of reports have described intralabyrinthine schwannomas. ${ }^{10}$

MRI with gadolinium is the best diagnostic tool to identify this disease. Intracochlear schwannomas may have slightly higher signal intensities than normal intralabyrinthine fluid on unenhanced T1-weighted images. On T2-weighted images, the schwannomas appear as hypointense lesions with sharp borders, and the fluid has a high signal. After gadolinium administration, the schwannomas present as strongly enhanced, sharply circumscribed lesions on T1-weighted images. ${ }^{11}$

Asymmetric sensorineural hearing loss is almost invariably present in patients with intracochlear schwannoma. Tinnitus, ear fullness, and vertigo are also common. Intracochlear schwannomas are difficult to diagnose, and diagnosis is often delayed because the presenting symptoms overlap with other otologic diseases (particularly Ménière's disease). Sometimes, patients present with mixed hearing loss and the conductive component can be secondary to stapes movement interference caused by tumor pressure on the vestibular surface of the stapes footplate or secondary to endolymphatic hydrops. ${ }^{12,13}$

Other lesions can mimic ILS on contrast-enhanced MRIs, including labyrinthitis (typically of viral etiology), labyrinthitis ossificans, hemorrhage, and lipoma. ${ }^{6}$

\section{Conclusion}

Treatment should preserve hearing and rehabilitate the patient. This minimally invasive procedure allows for simultaneous tumor removal and cochlear implantation with good audiological results. To the best of our knowledge, this report is the first description of simultaneous intracochlear schwannoma resection and cochlear implantation.
With this technique, we accessed the apical turn of the cochlea (with minimal damage to other structures) and removed the tumor. Thus, the intracochlear structure was preserved, which facilitated successful cochlear implantation and hearing restoration.

\section{References}

1 Bento RF. Tratado de Implante Coclear e Próteses Auditivas Implantáveis. 1st ed. Rio de Janeiro, Brazil: Thieme Publicações Ltda; 2014

2 von Ilberg C, Kiefer J, Tillein J, et al. Electric-acoustic stimulation of the auditory system. New technology for severe hearing loss. ORLJ Otorhinolaryngol Relat Spec 1999;61(6):334-340

3 Skarzyński H, Lorens A, D'Haese P, et al. Preservation of residual hearing in children and post-lingually deafened adults after cochlear implantation: an initial study. ORL J Otorhinolaryngol Relat Spec 2002;64(4):247-253

4 Bento RF, Brito Neto R, Castilho AM, et al. Resultados auditivos com o implante coclear multicanal em pacientes submetidos a cirurgia no Hospital das Clínicas da Faculdade de Medicina da Universidade de São Paulo. Braz J Otorhinolaryngol 2004;70:632-637

5 Kiefer J, Pok M, Adunka O, et al. Combined electric and acoustic stimulation of the auditory system: results of a clinical study. Audiol Neurootol 2005;10(3):134-144

6 Salzman KL, Childs AM, Davidson HC, Kennedy RJ, Shelton C, Harnsberger HR. Intralabyrinthine schwannomas: imaging diagnosis and classification. AJNR Am J Neuroradiol 2012;33(1): 104-109

7 Miller ME, Moriarty JM, Linetsky M, Lai C, Ishiyama A. Intracochlear schwannoma presenting as diffuse cochlear enhancement: diagnostic challenges of a rare cause of deafness. Ir J Med Sci 2012;181(1):131-134

8 Jiang ZY, Kutz JW Jr, Roland PS, Isaacson B. Intracochlear schwannomas confined to the otic capsule. Otol Neurotol 2011;32(7): $1175-1179$

9 Kronenberg J, Horowitz Z, Hildesheimer M. Intracochlear schwannoma and cochlear implantation. Ann Otol Rhinol Laryngol 1999; 108(7 Pt 1):659-660

10 Neff BA, Willcox TO Jr, Sataloff RT. Intralabyrinthine schwannomas. Otol Neurotol 2003;24(2):299-307

11 Magliulo G, Colicchio G, Romana AF, Stasolla A. Intracochlear schwannoma. Skull Base 2010;20(2):115-118

12 Green JD Jr, McKenzie JD. Diagnosis and management of intralabyrinthine schwannomas. Laryngoscope 1999;109(10): 1626-1631

13 Kennedy RJ, Shelton C, Salzman KL, Davidson HC, Harnsberger HR. Intralabyrinthine schwannomas: diagnosis, management, and a new classification system. Otol Neurotol 2004;25(2): 160-167 